\title{
Reconstrucción de defectos mandibulares: bloque acrílico como mantenedor de contorno de tejidos.
}

\section{Mandibular defects reconstruction: The use of an acrylic block as a space and contour maintainer of the oral tissues.}

\author{
Ignacio Araya ${ }^{1}$, Loreto Canto ${ }^{1}$, Gabriel Zamorano ${ }^{1}$, Nicolás Yanine ${ }^{1}$, Stefan Domancic ${ }^{1}$, Julio Villanueva ${ }^{1 *}$
}

\section{Departamento de Cirugía y Traumatología Bucal y Maxilofacial. Facultad de Odontología. Universidad de Chile. Chile}

* Correspondencia autor. Julio Villanueva| Sergio Livingstone Pohlhammer 943, Independencia, Santiago, Chile | Teléfono: +56229781841| E-mail: javm@uchile.cl

Trabajo recibido el 26/05/2018.

Aprobado para su publicación el 05/07/2018

\begin{abstract}
RESUMEN
Introducción: El tratamiento de grandes tumores mandibulares conlleva una alteración anatómica y funcional del paciente. La reconstrucción de estos defectos es un desafío para el cirujano, sin perjuicio que el tratamiento con injertos suele ser la primera opción en la mayoría de los casos, su aplicación podría estar limitada a las consecuencias derivadas de la comunicación del injerto con el medio oral. El presente artículo pretende exponer el uso de acrílico dental como elemento intermediario entre la resección y reconstrucción en situaciones como la descrita. Caso Clínico: Paciente adulta con ameloblastoma mandibular es tratada mediante resección quirúrgica y mantenida con un bloque acrílico en la zona resecada durante 6 meses, con el objeto de permitir el cierre de comunicación de la lesión con el medio oral, reconstruyéndose posteriormente el defecto con injerto libre de cresta ilíaca. Discusión: Las implicancias estético-funcionales de la mandíbula requieren meticulosidad en la reconstrucción de los defectos resultantes a su patología. A través del procedimiento propuesto, se consigue mejorar el pronóstico de la reconstrucción con injerto óseo de un defecto mandibular comunicado al medio oral, obteniéndose buenos resultados morfológicos y funcionales.
\end{abstract}

PALABRAS CLAVE:

Reconstrucción mandibular, Cirugía maxilofacial, Injerto óseo.

Rev. Clin. Periodoncia Implantol. Rehabil. Oral Vol. 12(1); 47-49, 2019.

\section{ABSTRACT}

Introduction: The treatment of large mandibular tumors has anatomic and functional sequelae to the patient. Reconstruction of these defects is a challenge to the surgeon, whereas grafting is the first choice treatment in most cases, its application could be limited by conditions such as a communication with the oral environment. The present article intends to present the use of a methacrylate block as an interim device between resection and reconstruction in situations such as the above described. Case Report: An adult female affected by mandibular ameloblastoma is treated by surgical resection and kept with the aid of a methacrylate block in the resected area for 6 months, allowing closure of the communication between the lesion and the oral environment to be posteriorly treated with a free iliac crest graft. Discussion: Functional and esthetic implications of mandibular resection sequelae mandate a thorough reconstruction of the lasting defects. By the proposed procedure a better prognosis for the reconstruction is achieved when using a bone graft in the setting of a lesion communicated with the oral environment, allowing good morphological and functional results.

KEY WORDS:

Mandible reconstruction; Maxillofacial surgery; Bone graft.

Rev. Clin. Periodoncia Implantol. Rehabil. Oral Vol. 12(1); 47-49, 2019

\section{INTRODUCCIÓN:}

El tratamiento de grandes tumores mandibulares $u$ otras patologías agresivas puede llevar a la resección en bloque, implicando la interrupción de inserciones musculares y la pérdida de inervación tanto sensitiva como motora, generando finalmente un impacto funcional en la masticación, deglución y fonoarticulación. Situación que pone de manifiesto la necesidad de realizar la reconstrucción mandibular.

Entre las alternativas existentes para la reconstrucción de la mandíbula están los injertos libres microvascularizados y no vascularizados, el más utilizado dentro de la última categoría es el de cresta ilíaca ${ }^{(1,2)}$. El tratamiento señalado se puede realizar de manera inmediata o diferida, teniendo en cuenta tanto las condiciones locales como la exposición a la cavidad oral, además de existir un riesgo significativamente mayor de complicaciones infecciosas posterior a la reconstrucción ${ }^{(1)}$, peligrando como consecuencia el injerto y con ello todo el procedimiento reconstructivo.

El polimetilmetacrilato o acrílico es ampliamente utilizado en odontología, al ser un material no degradable, biocompatible y fácilmente moldeable. Dentro de la gama de usos que se le ha dado, destaca su utilización en cirugía reconstructiva del territorio cráneofacial- en especial para la corrección de defectos en los tercios medio y superior del rostro, con buenos resultados estéticos y funcionales- además de ventajas desde el punto de vista quirúrgico ${ }^{(3-6)}$. Sin embargo, en reconstrucciones mandibulares son escasos los reportes de la aplicación de este material, salvo como mantenedor 
de espacio en la zona articular luego de cirugías correctoras de anquilosis temporomandibular ${ }^{(7)}$

El propósito de este artículo es dar a conocer el uso del acrílico en situaciones de reconstrucción de defectos mandibulares como mantenedor de espacio, morfología y función de los tejidos blandos perimandibulares.

\section{CASO CLÍNICO}

En julio de 2014 se presenta en el Servicio de Cirugía Maxilofacial del Hospital Clínico San Borja Arriarán una paciente de sexo femenino, 46 años, con un aumento de volumen mandibular derecho comunicado a boca a través de una perforación de la mucosa generada hace tres años al momento de la extracción del tercer molar (Figura 1 y 2). Al examen imagenológico (Radiografía y Tomografía) presenta una gran zona radiolúcida que compromete cuerpo y rama mandibular (Figura 3).

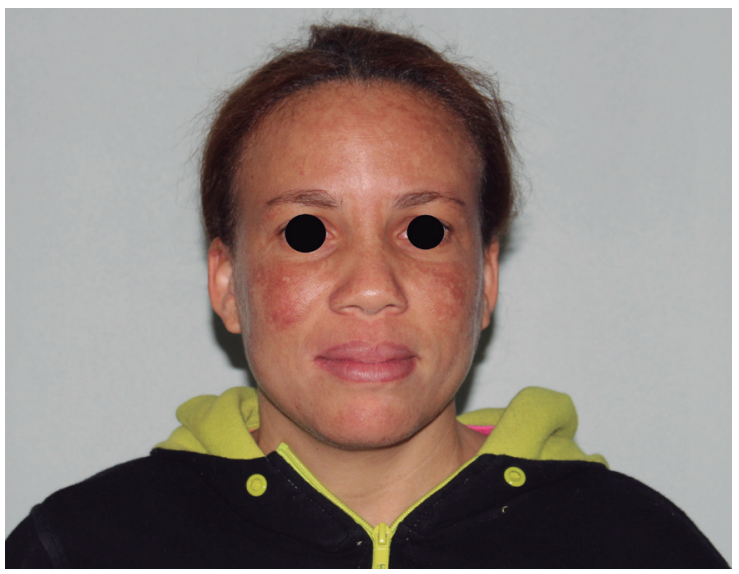

Figura 1. Foto clínica preoperatoria

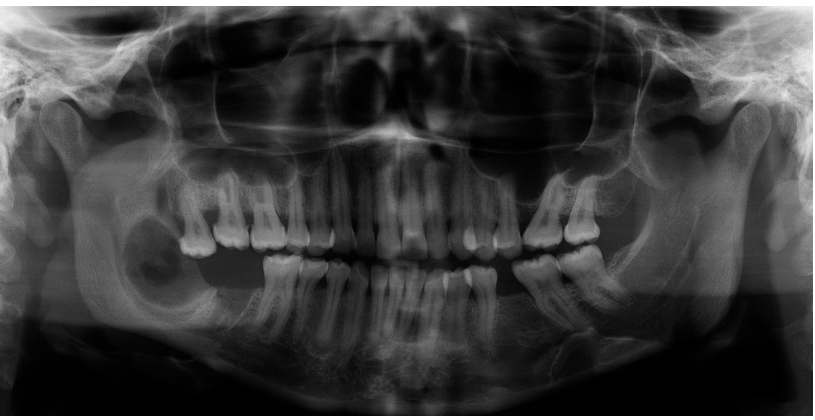

Figura 3. Radiografía Panorámica, que muestra la lesión que compromete cuerpo y rama mandibular

La biopsia reveló ameloblastoma sólido mandibular, un tumor odontogénico benigno ubicado en la mayoría de los casos en la mandíbula ${ }^{(8)}$. El tratamiento corresponde a la resección de los segmentos óseos comprometidos con margen de seguridad, dada su alta tasa de recidiva $a^{(9,10)}$.

En el caso descrito se indicó la resección en bloque de la lesión con márgenes de seguridad mediante un acceso quirúrgico extraoral. Para la planificación se utilizó un modelo estereolitográfico, el cual sirvió para la adaptación de una placa de reconstrucción y para la confección de un bloque de acrílico con la forma del segmento mandibular a resecar. Éste fue fabricado mediante la toma de impresión con alginato en la estereolitografía, realizándose posteriormente un vaciado con cera rosada para obtener como resultado final el bloque acrílico de termocurado, el cual incluyó en su mezcla gentamicina al $1 \%$.

Durante el intraoperatorio el bloque es fijado a la placa de reconstrucción utilizando tornillos de osteosíntesis (Figura 4), con el fin de dar sostén a los tejidos blandos, conservando un contorno anatómico adecuado y manteniendo el espacio para la colocación posterior del injerto óseo.

Ocho meses más tarde se retiró el bloque acrílico para realizar el injerto con cresta ilíaca, sirviendo este mismo para la toma del injerto y la consecución de la forma adecuada del mismo (Figura 5)

Actualmente, luego de 12 meses desde la primera cirugía (figuras 6 y 7), la paciente se encuentra en buenas condiciones, sin haber presentado complicaciones durante el tiempo de permanencia del bloque acrílico, con una morfología y dinámica mandibular conservadas, tanto durante el uso del mismo como con posterioridad a éste, en espera de la última intervención en la cual se planifica el retiro de la placa de reconstrucción una vez alcanzada una adecuada integración del injerto al hueso mandibular.

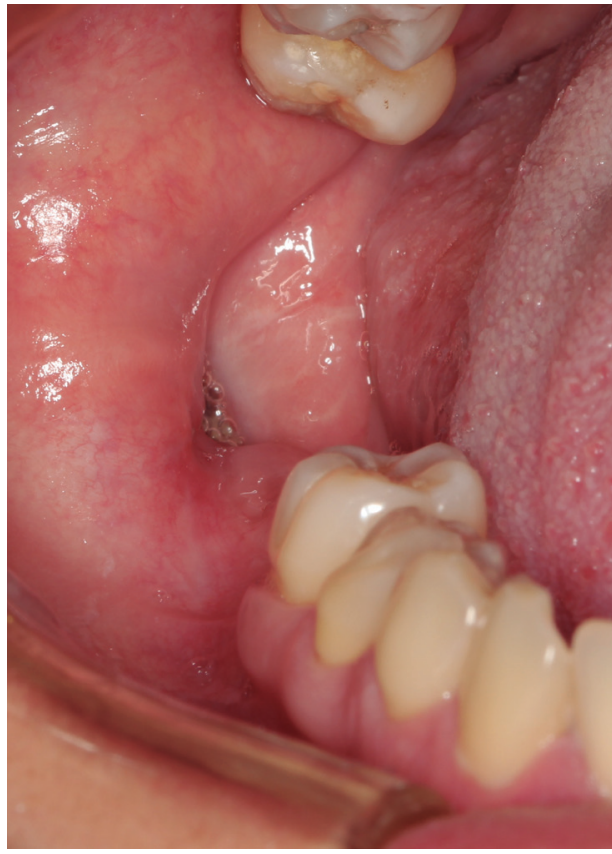

Figura 2. Vista intraoral de la lesión

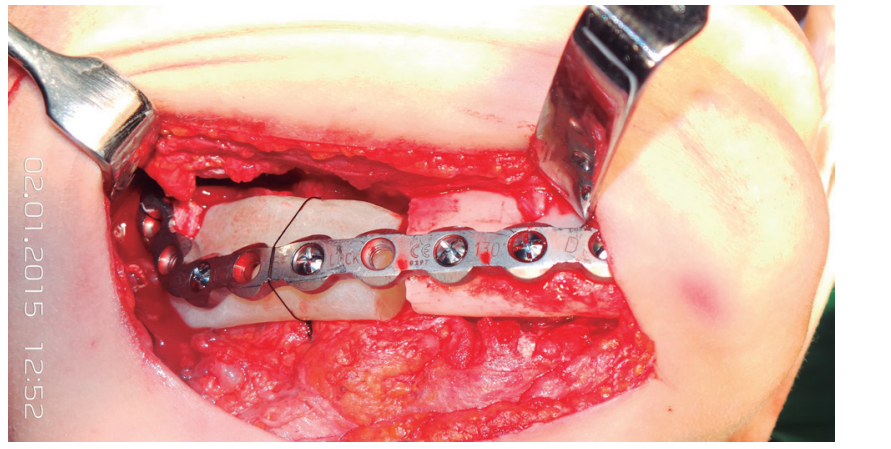

Figura 4. Placa acrílica fijada a la placa de reconstrucción

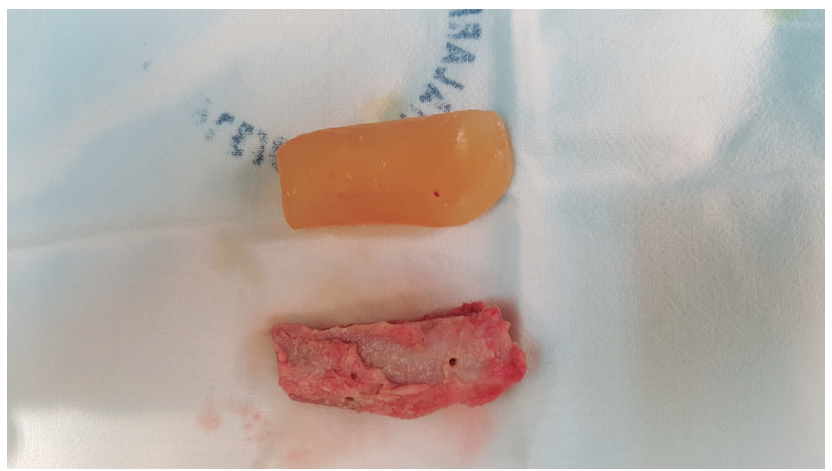

Figura 5. Injerto tallado según la guía acrilica

\section{DISCUSIÓN}

El ameloblastoma posee 4 tipos histológicos: i) sólido/multiquístico; ii) extraóseo/periférico; iii) desmoplástico y iv) uniquístico. El ameloblastoma sólido/multiquístico y uniquístico de subtipo folicular representan las variedades más agresivas clínicamente, recomendándose la resección y reconstrucción ${ }^{(11)}$.

La reconstrucción de defectos mandibulares post-cirugía resectiva es uno de los mayores retos de la cirugía maxilofacial. Son factores relevantes en la toma de decisiones la condición arquitectural mandibular y su constante sometimiento a fuerzas musculares que generan la dinámica mandibular y funciones como la deglución, masticación y fonoarticulación, además de la relevancia estética que reviste para el paciente.

Aunque actualmente la reconstrucción mandibular inmediata es la tendencia mundial, ésta fue materia controversial durante largo tiempo. Las técnicas de reconstrucción mandibular han variado enormemente desde su inicio en 1946. 


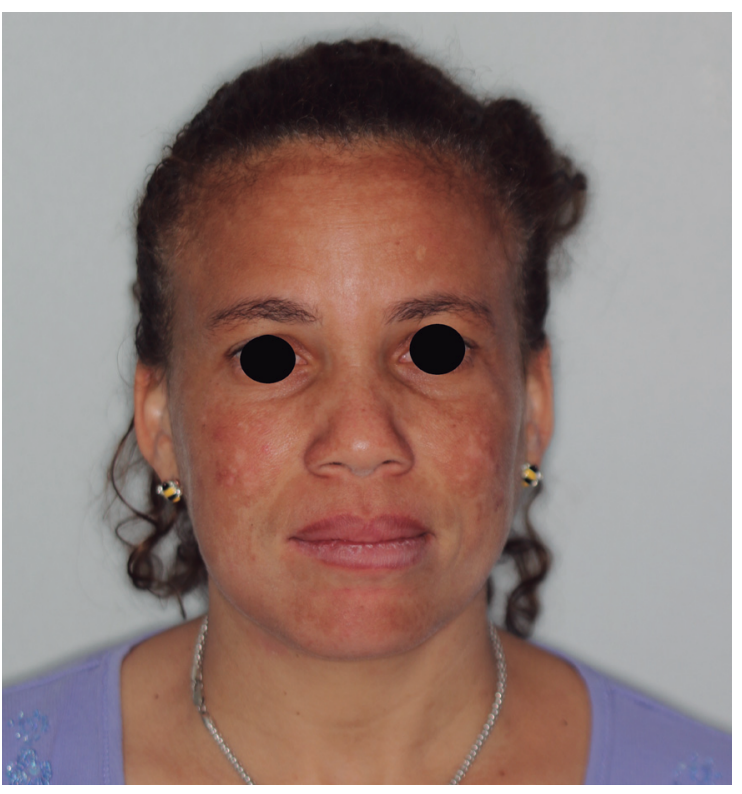

Figura 6. Foto clínica postoperatoria

Dentro de la diversidad de opciones disponibles actualmente podemos encontrar injertos miocutáneos; mio-osteo-cutáneos; óseos microvascularizados; óseos no vascularizados; placas de reconstrucción de titanio y materiales aloplásticos. Aun así, ningún método satisface todas las variables a las que está afecta una reconstrucción mandibular, siendo importante mencionar que no realizar reconstrucción alguna, es también una alternativa.

En la actualidad los injertos óseos libres microvascularizados y no vascularizados representan las alternativas de tratamiento más utilizadas, permitiendo una satisfactoria rehabilitación posterior, aunque-como todas las técnicas poseen indicaciones acotadas ${ }^{(12)}$.

No obstante, en la literatura internacional se describe muchas veces el uso de colgajos microvascularizados, en el contexto nacional esta alternativa representa varias limitaciones, dentro de ellas un alto costo, la coordinación de más de un equipo quirúrgico, una alta morbilidad del sitio dador $y$, aun así, su utilización no asegura el éxito de la reconstrucción. Por otra parte, el injerto óseo no vascularizado es de menor costo, morbilidad y posee la ventaja de tener un éxito similar sin comprometer profundamente al sitio dador, aunque sus indicaciones son más acotadas ${ }^{(12)}$

En esta nota técnica se expone un procedimiento sencillo que permite la reconstrucción inmediata, usando un material de bajo costo y de probada utilidad en reconstrucción, biocompatible y de baja toxicidad, ofreciendo un método sencillo y ventajoso para el paciente, teniendo presente además que conserva el contorno en virtud del rol de soporte que entrega a los tejidos blandos alrededor de la zona afectada, manteniendo una apariencia normal y permitiendo al paciente preservar la función mandibular a pesar de haber interrumpido su integridad estructural, mejorando el pronóstico de la reconstrucción definitiva.

Este material en reconstrucción craneal y de tercio medio, es utilizado como una alternativa terapéutica definitiva a los injertos óseos, en general de origen autólogo, considerados como el gold estándar ${ }^{(3)}$. En

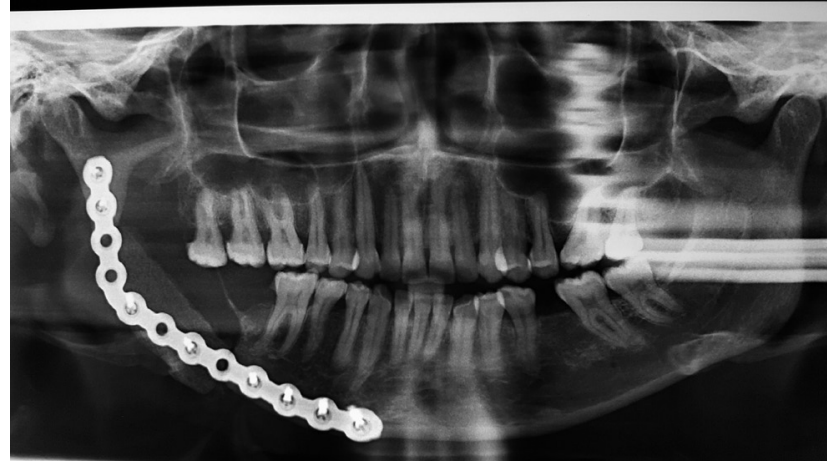

Figura 7. Control radiográfico del injerto.

cambio, en el procedimiento propuesto, posterior a la resección del tejido mandibular afectado con el margen de seguridad adecuado y luego de instalada la placa de reconstrucción, el bloque de acrílico es utilizado en forma transitoria, con el fin de evitar el colapso de los tejidos blandos alrededor de la placa.

En el contexto de la reconstrucción mandibular, no es de conocimiento de los autores, el uso de bloques acrílicos como elemento de reconstrucción reportado en la literatura, por lo que la técnica aquí descrita representa una aplicación novedosa de una técnica conocida y probada en otros contextos. Mantener la forma y con ello mejorar el confort del paciente desde la autopercepción, interacción social y recuperar la apariencia estética normal en casos de lesiones que alteren los contornos faciales, son objetivos deseables y alcanzables con este método.

Otras alternativas cercanas a las descritas en la literatura corresponden a implantes customizados, dentro de los cuales su composición y manejo es variable, viéndose también sujetos a una tasa de complicaciones significativa e implicando un mayor costo económico que debe sortear el paciente(13).

Es importante mencionar que en este caso se optó por la realización del procedimiento descrito atendida la interrupción de continuidad que presentaba la mucosa oral por el lapso de 3 años en el sitio, poniendo con esto en riesgo la viabilidad de un injerto inmediato, ejecutándose un paso intermedio con el fin de reducir esta probabilidad. Así, finalmente es posible realizar una cirugía reconstructiva con injertos óseos que permita la formación de nuevo hueso que devuelva la continuidad a la arquitectura mandibular, sirviendo el mismo bloque de acrílico como una guía para la toma y modelamiento del injerto, ayudando de esa forma a reducir la morbilidad del sitio dador, al no tener que extraer más tejido del necesario.

Una posible desventaja a considerar en la aplicación del procedimiento puede ser la necesidad de la obtención de modelos estereolitográficos para la óptima ejecución de éste, mas hoy en día estos no implican per se un difícil acceso ni costos muy elevados.

Finalmente, el procedimiento propuesto constituye una forma segura, sencilla y de relativo bajo costo para apoyar el proceso de reconstrucción de defectos mandibulares causados por cirugías resectivas como tratamiento de patologías óseas agresivas locales.

\section{CONFLICTO DE INTERÉS}

Los autores del trabajo declaran no tener conflicto de intereses.

\section{Bibliografía}

1. Van Gemert JTM, van Es RJJ, Van Cann EM, Koole R. Nonvascularized bone grafts for segmental reconstruction of the mandible--a reappraisal. J Oral Maxillofac Surg. 2009;67(7):1446-1152

2. Soto Góngora S, Texis González MG. Injertos óseos: Una alternativa efectiva y actual para la reconstrucción del complejo cráneo-facial. Rev Cuba Estomatol. 2005;42(1):0-0.

3. Lee SC, Wu CT, Lee, ST \& Chen PJ. Cranioplasty using polymethyl methacrylate prostheses. J Clin Neurosci. 2009;16(1)56-63.

4. Fernandes da Silva AL, Borba AM, Simão NR, Pedro FLM, Borges AH, Miloro M. Customized polymethyl methacrylate implants for the reconstruction of craniofacia osseous defects. Case Rep Surg. 2014;2014:358569

5. Lustica I, Velepic M, Cvjetković N, Bonifacić M, Kirincić N, Juretić M, et al. Polymethyl-methacrylate implants in forehead and supraorbital arches reconstruction: retrospective study. Coll Antropol. 2001;25 Suppl:137-43.

6. Abdo Filho RC, Oliveira TM, Lourenço Neto N, Gurgel C \& Abdo RC Reconstruction of bony facial contour deficiencies with polymethylmethacrylate implants: case report. J Appl Oral Sci. 2001;19(4)426-430.
7. Sawhney CP. Bony ankylosis of the temporomandibular joint: follow-up of 70 patients treated with arthroplasty and acrylic spacer interposition. Plast Reconstr Surg. 1986;77(1)29-40.

8. Masthan KMK, Anitha N, Krupaa J, Manikkam S. Ameloblastoma. J Pharm Bioallied Sci. 2015;7(Suppl 1):S167-70.

9. Bianchi B, Ferri A, Ferrari S, Leporati M, Copelli C, Ferri T, et al. Mandibular resection and reconstruction in the management of extensive ameloblastoma. J Oral Maxillofac Surg. 2013;71(3)528-537.

10. Tamme T, Tiigimäe J, Leibur E. Mandibular ameloblastoma: a 28-years retrospective study of the surgical treatment results. Minerva Stomatol. 2010;59(11 12)637-643

11. Haq J, Siddiqui S, McGurk M. Argument for the conservative management of mandibular ameloblastomas. Br J Oral Maxillofac Surg. 2016;54(9):1001-1005.

12. Kumar BP, Venkatesh V, Kumar K, Yadav B, Mohan SR. Mandibular Reconstruction: Overview. J. Maxillofac. Oral Surg. 2016;15(4):425-441.

13. Samman N, Cheung LK, Tideman H. Functional reconstruction of the jaws: new concepts. Ann R Australas Coll Dent Surg. 1996;13:184-192. 